\title{
In vivo measurement of the frame-based application accuracy of the Neuromate neurosurgical robot
}

\author{
Daniel von Langsdorff, MD,' Philippe Paquis, MD, ${ }^{1}$ and Denys Fontaine, MD, PhD1,2 \\ 1'Department of Neurosurgery, Centre Hospitalier Universitaire de Nice; and ${ }^{2}$ IGCN-EA 7282 (Image-Guided Clinical \\ Neuroscience and Connectomics), UMR 6284 ISIT, Université d'Auvergne, Clermont-Ferrand, France
}

\begin{abstract}
OBJECT The application accuracy of the Neuromate neurosurgical robot has been validated in vitro but has not been evaluated in vivo for deep brain stimulation (DBS) electrode implantations. The authors conducted a study to evaluate this application accuracy in routine frame-based DBS procedures, with an independent system of measurement.

METHODS The Euclidian distance was measured between the point theoretically targeted by the robot and the point actually reached, based on their respective stereotactic coordinates. The coordinates of the theoretical target were given by the robot's dedicated targeting software. The coordinates of the point actually reached were recalculated using the Stereoplan localizer system. This experiment was performed in vitro, with the frame fixed in the robot space without a patient, for 21 points spatially distributed. The in vivo accuracy was then measured in 30 basal ganglia targets in 17 consecutive patients undergoing DBS for movement disorders.

RESULTS The mean in vitro application accuracy was $0.44 \pm 0.23 \mathrm{~mm}$. The maximal localization error was $1.0 \mathrm{~mm}$. The mean $( \pm \mathrm{SD})$ in vivo application accuracy was $0.86 \pm 0.32 \mathrm{~mm}(\Delta \mathrm{x}=0.37 \pm 0.34 \mathrm{~mm}, \Delta \mathrm{y}=0.32 \pm 0.24 \mathrm{~mm}, \Delta \mathrm{z}=$ $0.58 \pm 0.31 \mathrm{~mm})$. The maximal error was $1.55 \mathrm{~mm}$.

CONCLUSIONS The in vivo application accuracy of the Neuromate neurosurgical robot, measured with a system independent from the robot, in frame-based DBS procedures was better than $1 \mathrm{~mm}$. This accuracy is at least similar to the accuracy of stereotactic frame arms and is compatible with the accuracy required in DBS procedures.

http://thejns.org/doi/abs/10.3171/2014.9.JNS14256
\end{abstract}

KEY WORDS accuracy; deep brain stimulation; Neuromate robot; stereotactic frame; functional neurosurgery

$\mathrm{R}$ овотіс neurosurgery has been developed for nearly 25 years and offers neurosurgeons many advantages, especially increased accuracy. ${ }^{10}$ Accuracy is a key point for stereotactic neurosurgical procedures, particularly for implantations of deep brain stimulation (DBS) electrodes. The Neuromate robot (Renishaw) is a commercially available neurosurgical robot used in many centers around the world for stereotactic and endoscopic procedures (Fig. 1A). According to the manufacturer, its accuracy is better than $1 \mathrm{~mm}$ and its reproducibility is $0.15 \mathrm{~mm}$, leading to maximal localization errors of less than $2 \mathrm{~mm} \cdot{ }^{13}$ However, no data are available in the literature concerning its application accuracy measured in vivo, during DBS procedures, although its frame-based and frameless accuracies have been measured in vitro ${ }^{8,13}$ and during stereoelectroencephalography (SEEG) procedures. ${ }^{1}$

The Neuromate robot was recently acquired in our center (Centre Hospitalier Universitaire de Nice, France), and we wanted to evaluate its actual application accuracy in routine frame-based DBS procedures with a system of measurement independent from the robot itself and from the manufacturer.

\section{Methods}

The objective of this study was to quantify the application accuracy of the Neuromate robot during frame-based DBS procedures, by comparing the distance between the point theoretically targeted by the robot and the point actually reached, based on their respective stereotactic coordinates. The stereotactic coordinates of the theoretical target were given by the robot's dedicated targeting software (VoXim, IVS Technology GmbH). The stereotactic frame-based coordinates of the actual point reached were recalculated using the Stereoplan localizer system (Fig. 

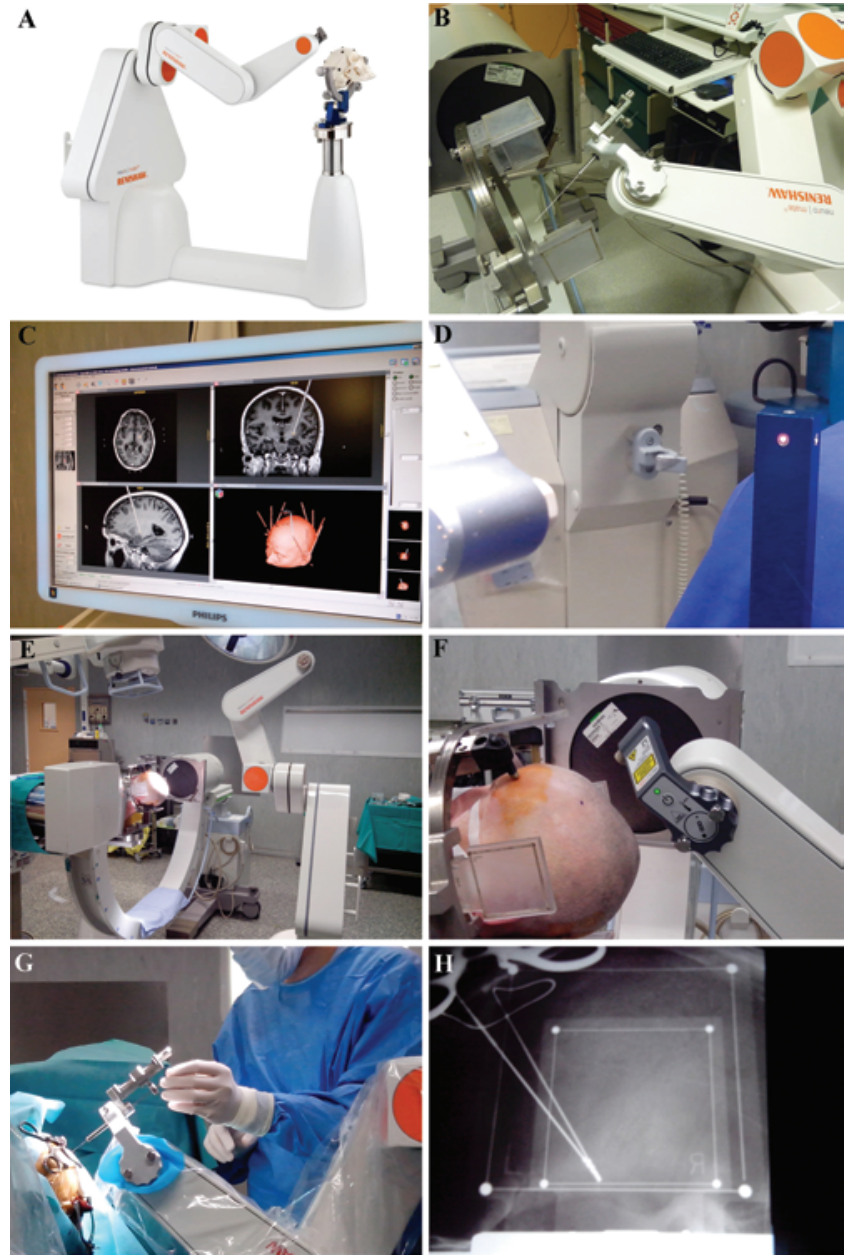

FIG. 1. Measurement of the Neuromate robot application accuracy using the Stereoplan system (A and B), and workflow of a DBS procedure using the robot and in vivo measurement of its accuracy $(\mathrm{C}-\mathrm{H})$. A: Overall view of the Neuromate robot. B: In vitro measurement. Four localizing small plates were fixed on the Fischer frame, each of them containing radiopaque fiducials arranged in a square. The robotic arm was brought to the trajectory in the target position, and a rigid tube was introduced to the trajectory. Anteroposterior and lateral radiographs were obtained to identify these fiducials and calculate the location of the tip of the guiding tube according to the fiducial locations. C: Target definition and trajectory planning using the VoXim software. D: Preoperative checking of robot calibration by projection of a laser beam onto the center of the dedicated checking device. E: The Fischer frame was fixed on the patient's head and then fixed in the robot head holder in a uniquely defined position, allowing the direct automatic registration of the frame to the robot. F: The localizing small plates were then fixed on the frame. The laser beam mounted on the robotic arm indicated the trajectory entry point. G: Using the microdrive, a rigid guiding tube was introduced with the guidance of the robotic arm following the defined trajectory to the target. H: Lateral intraoperative radiograph showing the projection of the fiducials of the left and right localizing small plates and the guiding tube inserted to the target. The DBS electrode on the contralateral side is also visible. Figure is available in color online only.

1B) and software (Stereoplan version 2.0, Leibinger), a system used for years in routine DBS procedures., ${ }^{3,5-7,12}$

\section{Planning and Targeting Procedure}

After head fixation of a stereotactic titanium Fischer frame, 3D T1-weighted MRI was performed. The images and frame fiducials were then registered by the robot's VoXim planning software (Fig. 1C). The anatomical target and trajectory were defined and the stereotactic coordinates of this theoretical target $\left(\mathrm{x}_{\mathrm{T}}, \mathrm{y}_{\mathrm{T}}, \mathrm{z}_{\mathrm{T}}\right)$ were given by the software. Calibration of the Neuromate robot was checked before the surgery by projection of a laser beam using the dedicated checking device (Fig. 1D).

The patient was then positioned with the head and frame fixed in the robot frame holder, allowing the automatic mechanical registration of the frame to the robot (Fig. 1E). The entry point was located using the laser beam (Fig. 1F). Through a bur hole, a rigid guiding tube was introduced with the guidance of the robotic arm following the defined trajectory to the target (Fig. 1G).

\section{Stereotactic Coordinates Verification Procedure}

Calculation of the intraoperative real stereotactic coordinates $\left(\mathrm{x}_{\mathrm{R}}, \mathrm{y}_{\mathrm{R}}, \mathrm{z}_{\mathrm{R}}\right)$ of the tip of the guiding tube was performed using the Stereoplan 2.0 digitizing system, specific to the Fischer frame. Four fiducial small plates were fixed on the frame (Fig. 1B and F), each of them containing 4 radiopaque fiducials arranged in a square. After introduction of the guiding tube to the target through the microdrive, anteroposterior and lateral radiographs were obtained and digitized, allowing recognition of the fiducials and calculation of the magnification and deformation of the squares by the software (Fig. 1H). The Stereoplan software then calculated the actual Fischer frame-based stereotactic coordinates of the tip of the guiding tube by calculating the spatial location of the tip of the guiding tube relative to the respective 2D locations of the 16 fiducials.

\section{Measurement of the Neuromate Application Accuracy}

As the coordinates of both theoretical and real targets were given in the same frame space, their Euclidian distance "d" was defined as the square root of the sum of the squares of the differences $(\Delta)$ between the corresponding coordinates of the points: $\mathrm{d}^{2}=\left(\mathrm{x}_{\mathrm{T}}-\mathrm{x}_{\mathrm{R}}\right)^{2}+\left(\mathrm{y}_{\mathrm{T}}-\mathrm{y}_{\mathrm{R}}\right)^{2}+\left(\mathrm{z}_{\mathrm{T}}\right.$ $\left.-z_{R}\right)^{2}$. This Euclidian distance defined the application accuracy of the robot.

In the first step, this experiment was performed in vitro, with the stereotactic frame fixed in the robot space without a patient. The overall accuracy measured in these experimental conditions was the sum of each of the following individual inaccuracies: localization error due to stereotactic frame deformation, accuracy of the frame registration in the robot space, mechanical accuracy of the robotic arm, accuracy of the microdrive, deformation of the guiding tube, and inaccuracy of the Stereoplan measuring system. Our method allows one to measure the robot's accuracy independently from the quality and/or distortion of the MR images and from the definition of the target on the images.

In the second step, this measurement was performed in a consecutive series of patients undergoing robot-guided frame-based DBS. The overall accuracy measured in these experimental conditions was the sum of the previously mentioned errors, plus the inaccuracy due to the potential guiding tube's bend within the head, plus any other localization error due to the patient's presence. 


\section{Results}

\section{In Vitro Measurements}

We defined 21 points distributed in the stereotactic space and reached them with the robotic arm through highly angulated trajectories. The accuracy at the central point of the frame was $0.37 \mathrm{~mm}(\Delta x=0.2 \mathrm{~mm}, \Delta y=0.3$ $\mathrm{mm}, \Delta \mathrm{z}=0 \mathrm{~mm})$. The mean $\pm \mathrm{SD}$ application accuracy for the 21 points was $\mathrm{d}=0.44 \pm 0.23 \mathrm{~mm}(\Delta \mathrm{x}=0.16 \pm$ $0.27 \mathrm{~mm}, \Delta y=0.3 \pm 0.24 \mathrm{~mm}, \Delta \mathrm{z}=0 \pm 0.21 \mathrm{~mm})$. The maximal localization error was equal to $1.0 \mathrm{~mm}$.

\section{In Vivo Measurements}

In vivo application accuracy was measured in 30 basal ganglia targets (13 globus pallidus internus, 10 subthalamic nucleus, 7 ventralis intermedius nucleus) in 17 consecutive patients undergoing DBS for movement disorders. In this small series, there were no complications and the outcome was similar to the outcome of patients who underwent operations using our previous frame-based procedure. The mean $\pm \mathrm{SD}$ application accuracy was $\mathrm{d}=$ $0.86 \pm 0.32 \mathrm{~mm}(\Delta \mathrm{x}=0.37 \pm 0.34 \mathrm{~mm}, \Delta \mathrm{y}=0.32 \pm 0.24$ $\mathrm{mm}, \Delta \mathrm{z}=0.58 \pm 0.31 \mathrm{~mm})$. The maximal localization error was equal to $1.55 \mathrm{~mm}(\Delta \mathrm{x} \max =1.3 \mathrm{~mm}, \Delta \mathrm{y} \max =$ $0.9 \mathrm{~mm}, \Delta \mathrm{z} \max =1.2 \mathrm{~mm})$. The accuracy did not differ across targets and patient diseases.

\section{Discussion}

In these routine frame-based DBS procedures, the application accuracy of the Neuromate robot, measured in vivo by a method independent from the robot, was better than $1 \mathrm{~mm}$. This accuracy was the sum of all individual inaccuracies, including the errors of the frame registration in the robot space, the mechanical inaccuracy of the robotic arm, the deformation of the guiding tube, and the inaccuracy of the Stereoplan measuring system. In the Neuromate robotic system, the frame is rigidly fixed to the robot in a uniquely defined position, resulting in a reliable mechanical robot-frame registration. The calibration of the robotic arm was checked before the beginning of the surgery. The precision (repeatability) of the robotic arm measured by our method in vitro was 0.23 $\mathrm{mm}$, very close to the $0.15-\mathrm{mm}$ precision claimed by the manufacturer. We localized the tip of a rigid guiding tube rather than the more flexible DBS electrode, to decrease inaccuracy related to its potential deformation within the brain. Ultimately, every measuring system has its own inaccuracy. Previous similar in vitro neurosurgical robot accuracy studies have used different measuring systems, including dedicated phantoms combined with mechanical measuring devices, ${ }^{8,9}$ infrared sensors, ${ }^{8,13}$ or Vernier calipers. ${ }^{4}$ These measuring devices are not adapted to in vivo measurements due to the inaccessibility of the needle's tip within the brain. Previous in vivo studies have used intraoperative CT scans to calculate the needle-target distance ${ }^{2}$ or a planned versus actual targets distance on merged CT scans. ${ }^{1}$ We used the Stereoplan system, a very accurate measuring system used routinely for many years to intraoperatively check the location of DBS electrodes. ${ }^{3,5-7,12}$ The Stereoplan requires orthogonal radiographs only, but requires the use of the Fischer stereotactic frame and fiducials. For this reason we were able to measure the in vivo accuracy of the Neuromate robot in frame-based conditions only, although its frameless utilization also has numerous advantages.

In both in vitro and in vivo conditions, the application accuracy of the Neuromate robot was better than $1 \mathrm{~mm}$. These results were concordant with previous similar results of in vitro studies. Using a Fischer frame and a dedicated phantom, Li et al. reported that the Neuromate application accuracy was $0.9 \mathrm{~mm} .{ }^{8}$ This was slightly higher than the $0.4 \mathrm{~mm}$ accuracy that we observed in vitro, but their results also included errors related to CT scans and target definition. Varma and Eldridge reported that the Neuromate accuracy measured with a phantom in frameless conditions was $1.3 \mathrm{~mm},{ }^{13}$ but frameless registration is known to be less accurate than frame-based registration. ${ }^{8}$ In vivo Neuromate application accuracy has only been evaluated during SEEG procedures by Cardinale et al. ${ }^{1}$ In that study, only the entry points of the bone screws were localized stereotactically with the robot, and the electrodes were then implanted under fluoroscopic guidance only. The distance between the planned and actual trajectories was calculated on the fused pre- and intraoperative CT scans, respectively. In 1050 SEEG electrodes implanted in 91 patients, the mean accuracy of the screw tip location was $0.9 \mathrm{~mm}$, which was similar to our results. However, the maximal error was $3.9 \mathrm{~mm}$, and the error was greater than $2 \mathrm{~mm}$ in $3 \%$ of the patients. This might be explained by aberrant screw trajectories in the bone, especially in cases of a trajectory tangent to the skull surface, and by the inaccuracy of the CT scan merging process. ${ }^{11}$

The submillimeter application accuracy of the Neuromate robot is at least equivalent to the accuracies of stereotactic frames classically used in DBS procedures. Applying the same Stereoplan methodology, the accuracy of the Fischer frame combined with the ZD arm was 0.9 $\mathrm{mm} .{ }^{14}$ Maciunas et al. have reported that the application accuracies of several stereotactic frames were 1.2-1.9 mm. ${ }^{9}$ Consequently, we believe that the application accuracy of the Neuromate that we evaluated in frame-based conditions is compatible with the accuracy required in DBS procedures.

\section{Conclusions}

The in vivo application accuracy of the Neuromate neurosurgical robot, measured with a system independent from the robot, in frame-based DBS procedures was better than $1 \mathrm{~mm}$. This accuracy is at least similar to the accuracy of stereotactic frame arms and is compatible with the accuracy required in DBS procedures.

\section{References}

1. Cardinale F, Cossu M, Castana L, Casaceli G, Schiariti MP, Miserocchi A, et al: Stereoelectroencephalography: surgical methodology, safety, and stereotactic application accuracy in 500 procedures. Neurosurgery 72:353-366, 2013

2. Chan F, Kassim I, Lo C, Ho CL, Low D, Ang BT, et al: Image-guided robotic neurosurgery - an in vitro and in vivo point accuracy evaluation experimental study. Surg Neurol 71:640-648, 2009 
3. Derrey S, Maltête D, Chastan N, Debono B, Proust F, Gérardin E, et al: Deep brain stimulation of the subthalamic nucleus in Parkinson's disease: usefulness of intraoperative radiological guidance. The Stereoplan. Stereotact Funct Neurosurg 86:351-358, 2008

4. Eljamel MS: Validation of the PathFinder neurosurgical robot using a phantom. Int J Med Robot 3:372-377, 2007

5. Fontaine D, Borg M, Bayreuther C, von Langsdorff D, Magnie-Mauro MN, Chanalet S, et al: [Subthalamic nucleus stimulation using a Fisher ZD stereotactic frame, MR-CT fusion guidance and peroperative orthogonal radiographs, in Parkinson disease.] Neurochirurgie 53:463-469, 2007 (Fr)

6. Hamel W, Fietzek U, Morsnowski A, Schrader B, Herzog J, Weinert D, et al: Deep brain stimulation of the subthalamic nucleus in Parkinson's disease: evaluation of active electrode contacts. J Neurol Neurosurg Psychiatry 74:1036-1046, 2003

7. Hamel W, Schrader B, Weinert D, Herzog J, Volkmann J, Deuschl G, et al: MRI- and skull x-ray-based approaches to evaluate the position of deep brain stimulation electrode contacts - a technical note. Zentralbl Neurochir 63:65-69, 2002

8. Li QH, Zamorano L, Pandya A, Perez R, Gong J, Diaz F: The application accuracy of the NeuroMate robot-a quantitative comparison with frameless and frame-based surgical localization systems. Comput Aided Surg 7:90-98, 2002

9. Maciunas RJ, Galloway RL Jr, Latimer JW: The application accuracy of stereotactic frames. Neurosurgery 35:682-695, 1994

10. McBeth PB, Louw DF, Rizun PR, Sutherland GR: Robotics in neurosurgery. Am J Surg 188 (4A Suppl):68S-75S, 2004
11. Schicho K, Figl M, Seemann R, Ewers R, Lambrecht JT, Wagner A, et al: Accuracy of treatment planning based on stereolithography in computer assisted surgery. Med Phys 33:3408-3417, 2006

12. Schrader B, Hamel W, Weinert D, Mehdorn HM: Documentation of electrode localization. Mov Disord 17 (Suppl 3):S167-S174, 2002

13. Varma TR, Eldridge P: Use of the NeuroMate stereotactic robot in a frameless mode for functional neurosurgery. Int $\mathbf{J}$ Med Robot 2:107-113, 2006

14. von Langsdorff D, Fontaine D, Niesar E, Chanalet S, Raucoules S, Paquis P: A simple and reproducible method to check the accuracy of your stereotactic systems and procedures. Stereotact Funct Neurosurg 76:184, 2001 (Abstract)

\section{Author Contributions}

Conception and design: Fontaine, Paquis. Acquisition of data: Fontaine, von Langsdorff. Analysis and interpretation of data: Fontaine, von Langsdorff. Drafting the article: Fontaine, von Langsdorff. Critically revising the article: Fontaine, von Langsdorff. Reviewed submitted version of manuscript: all authors. Approved the final version of the manuscript on behalf of all authors: Fontaine. Administrative/technical/material support: Fontaine, Paquis. Study supervision: Fontaine, Paquis.

\section{Correspondence}

Denys Fontaine, Service de Neurochirurgie, Hôpital Pasteur, 30 Avenue de la Voie Romaine, 06000 Nice, France. email: fontaine.d@chu-nice.fr. 